\title{
Pilot Decontamination in Massive MIMO Systems based on Pilot Design and Allocation
}

\author{
Jingwei Dong*, Zhiyu Han, and Chuang Han \\ School of Measure-Control Technology and Communication Engineering, Harbin University of Science and Technology, Harbin, 150080, China
}

\begin{abstract}
Pilot contamination has become an important factor affecting the development of massive MIMO. To mitigate pilot contamination, a new scheme of joint polar coordinate pilot assignment and pseudo random code pilot design is proposed in this paper. The scheme allocates the pilot according to the size of the user's polar angle, which is marked with polar coordinates, and uses pseudo random codes with different time delays to add disturbance to users that share the same pilot sequence and users of neighboring cells so as to improve the accuracy of channel estimation. Through theoretical derivation and data simulation analysis of minimum mean square error (MMSE) channel estimation of the system, the scheme can significantly reduce the mean square error (MSE) of channel estimation and improve the signal-to-noise ratio and average transmission rate of uplink transmission. Thus, the adverse effect of pilot contamination on channel estimation is reduced, and the channel throughput is improved.
\end{abstract}

Keywords: massive MIMO; pilot contamination; channel estimation; pilot sequence

(Submitted on April 1, 2019; Revised on May 15, 2019; Accepted on September 22, 2019)

(C) 2019 Totem Publisher, Inc. All rights reserved.

\section{Introduction}

In recent years, mobile data traffic has shown explosive growth with the increase in the number of mobile terminals. Some experts predict that data traffic in the future will be significantly higher than current data traffic, so it is increasingly difficult for the current communication system to meet people's demands for the connected intelligence of the future. 5G is a new generation communication system network that was proposed under this background [1]. As one of the keys of 5G, massive MIMO has been widely concerned by scholars because it can make full use of space resources, concentrate beams in a narrow range, and greatly improve power efficiency by increasing the number of antennas from transmitter to receiver to tens or hundreds of antennas. Marzetta found in 2010 that additive Gaussian noise and uncorrelated interference vanish with unlimited BS antennas, small-scale fading will be averaged out, and the channel vector between base stations and users will be gradual orthogonal [2].

For massive MIMO systems, accurate channel estimation is critical for achieving high capacity. In ideal conditions, the system should assign orthogonal pilot sequences to each user. Nonetheless, due to limitations by coherence time, the length of pilot sequences and the number of orthogonal pilots are finite; for example, the maximum number of orthogonal pilots in the channel coherent time of $1 \mathrm{~ms}$ is about 200 in the classic scenario, and non-orthogonal pilots must be utilized in multicell systems when the number of users is numerous. Subsequently, pilot sequences used by users will also be nonorthogonal or completely identical, which will lead to pilot contamination and reduce the accuracy of system channel state information estimation. Therefore, the exploration of pilot contamination plays an important role in improving the performance of massive MIMO systems.

At present, several methods have been proposed to reduce pilot contamination. The schemes for massive MIMO pilot pollution reduction are mainly divided into four categories [3-5]: optimized channel estimation method, precoding matrix method, pilot assignment scheme, and pilot sequence design. References [6-9] reduced the influence of pilot contamination

\footnotetext{
* Corresponding author.

E-mail address: djw@hrbust.edu.cn
} 
by improving the method of channel estimation. The authors proposed a method of constructing a channel autocorrelation matrix by using the exponential correlation model in [6]. The joint SVD-based method and iterative least square with projection (ILSP) algorithm was introduced in [7]. However, these blind algorithms were only employed in slowly varying channel scenarios and were impaired by scalar ambiguity. A massive MIMO channel estimation scheme based on beam domain decomposition and SVD was proposed in [9]. To optimize the method of precoding matrix, Gao solved the pilot pollution problem by using the ZF precoding algorithm based on Gauss-Seidel iteration in [10]. [11] used methods such as adjusting the time slot of the sending pilot by different users or conducting sector partitioning of cells to reduce the influence of pilot contamination on the system. To optimize pilot sequence design, a pilot design scheme of the ZC sequence, which did not need to carry out inverse calculation of variables, was proposed in [12].

In this paper, a scheme of joint polar coordinate pilot assignment and pseudo random code pilot design is proposed. First, the orientation of each user in the cell is marked by using polar coordinates, and the pilot is assigned according to the size of the user's polar angle. Then, the pseudo random codes with different time delays are used to disturb the users who use the same pilot sequence in neighbour cells. Compared with the traditional method, which only assigns the pilot to the same size of the user's polar angle in polar coordinates, the scheme in this paper can assign the pilot reasonably and further reduce the correlation of users using the same pilot in different cells. The system model has low computing complexity. In addition, compared with the tradition scheme, including hierarchical pilot allocation and integer pilot allocation, this scheme can save the number of orthogonal pilots used and more effectively improve the accuracy of channel estimation.

\section{System Modeling}

\subsection{The Channel Model}

In this paper, a massive MIMO multi-cell TDD cellular system is studied. It is composed of $L$ hexagonal cells, and each cell contains a base station equipped with $M$ directional antennas and $K$ single antenna users. All users share the same bandwidth resources. Assume the average power transmitted by the base station is $p_{f}$, and the average power transmitted by the user is $p_{r}$. The channel transmission vector from the $k^{\text {th }}$ user in cell $j$ to the $m^{\text {th }}$ antenna in cell $i$ is

$$
g_{i m j k}=h_{i m j k} \sqrt{\beta_{i j k}}
$$

Where $h_{\text {imjk }} \sim C N(0,1)$ is a small-scale fading vector between the user and the antenna, and $\sqrt{\beta_{i j k}}$ is the shadow fading and geometric fading vector between the user and the antenna.

$$
\beta_{i j k}=\frac{z_{i j k}}{\left(r_{i j k} / R\right)^{\alpha}}
$$

Where $Z_{i j k}$ is a lognormal random variable whose mean is 0 and variance is $1 . r_{i j k}$ is the distance from the $k^{\text {th }}$ user in the $j^{\text {th }}$ cell to the $i^{\text {th }}$ cell base station, $R$ is the radius of the cell, and $\alpha$ is the path loss coefficient.

\subsection{The Uplink Training}

In the massive MIMO multi-cell TDD cellular system, the base station uses the pilot uploaded by the users for channel estimation, completes the signal detection and downlink precoding through the results of channel estimation, and finally transmits the signal back to the users. Due to the large mutual interference between different users in the same cell, orthogonal pilot sequences are usually assigned to users in the cell.

$$
\Phi=\left[\varphi_{1}, \varphi_{2}, \varphi_{3}, \cdots, \varphi_{K}\right]^{\mathrm{T}} \in C^{K \times \tau}
$$

Where $\tau$ is the length of the pilot sequence and $\varphi_{K}$ is the pilot used by the $k^{\text {th }}$ user in the cell, which follows $\varphi_{K} \varphi_{K}^{H}=I$. In the uplink, the orthogonal pilot matrix sent by the cell base station to each cell user is 


$$
Y_{i}^{p}=\sqrt{p_{r}} \sum_{j=1}^{L} \sum_{k=1}^{K} g_{i j k} \varphi_{k}^{T}+N_{i}^{p}
$$

Where $N_{i}^{p}$ is an additive white Gaussian noise matrix, and $N_{i}^{p} \in C^{M \times \tau}$. It is usually assumed to be an independent isomorphic random variable with a mean of 0 and variance of 1.

After receiving the pilot signal, the base station uses it for channel estimation. Least square (LS) channel estimation is employed in this paper, and the result is

$$
\hat{g}_{i i k}=\frac{1}{\sqrt{p_{r}}} Y_{i}^{p}\left(I+p_{r} \tau \sum_{j=1}^{L} \sum_{k=1}^{K} \varphi_{k}^{*} \sqrt{\beta_{i j k}} \varphi_{k}^{H}\right)^{-1} \varphi_{i}^{*} \sqrt{\beta_{i k}}
$$

The channel estimation result between the $k^{\text {th }}$ user and the base station in the cell is the superposition of the channel transmission vectors between the user using the same pilot sequence and the target cell base station; thus, pilot contamination occurs [13].

After the channel estimation is completed, the base station receives the data signal uploaded by the user.

$$
Y_{i}^{u}=\sqrt{p_{r}} \sum_{j=1}^{L} \sum_{k=1}^{K} g_{i j k} x_{j k}^{u}+n_{i}^{u}
$$

Where $x_{j k}^{u}$ is the uplink data sent by the $k^{\text {th }}$ user in the $j^{\text {th }}$ cell, and $n_{i}^{u} \in C^{M \times 1}$ is a complex Gaussian noise whose mean is 0 and variance is 1 .

The base station then completes signal detection by the channel estimation result of Equation (4), and the user data received. In this paper, matched filter (MF) is employed to multiply the data signal received by the base station and the conjugate transpose of channel estimation. The signal detection of the $k^{\text {th }}$ user in the $i^{\text {th }}$ cell is

$$
\hat{x}_{i k}^{u}=\hat{g}_{i i k}^{H} Y_{i}^{u}=\left(\sum_{j=1}^{L} g_{i j k}+w_{i k}\right)^{H}\left(\sqrt{p_{r}} \sum_{j=1}^{L} \sum_{k=1}^{K} g_{i j k} x_{j k}^{u}+n_{i}^{u}\right)=\sqrt{p_{r}}\left(g_{i i k}^{H} g_{i i k} x_{i k}^{u}+\sum_{j \neq i} g_{i j k}^{H} g_{i j k} x_{i k}^{u}\right)+\varepsilon_{i k}^{u}
$$

From above, it can be concluded that the uplink signal to interference and noise ratio (SINR) of the $k^{\text {th }}$ user in the $i^{\text {th }}$ cell is

$$
S_{i k}^{u}=\frac{\left|g_{i i k}^{H} g_{i i k}\right|^{2}}{\sum_{j \neq i}\left|g_{i j k}^{H} g_{i j k}\right|^{2}+\frac{\left|\varepsilon_{i i k}^{u}\right|^{2}}{p_{r}}}
$$

With the number of cell base station antennas approaching infinity, the channel will be progressive orthogonal.

$$
\lim _{m \rightarrow \infty} S_{i k}^{u}=\frac{\beta_{i i k}^{2}}{\sum_{j \neq i} \beta_{i j k}^{2}}
$$

From above, it can be seen that when the number of antennas of the cell base station approaches infinity, the influence of small-scale fading coefficients and noise on the system will be negligible. The SINR of the uplink is only affected by the large-scale fading of the user, which allocates the same pilot sequence in each cell [14].

According to Equation (8) and the Shannon formula, the uplink reachable rate of the $k^{\text {th }}$ user in the $i^{\text {th }}$ cell is

$$
c_{i k}^{u}=\left(1-\mu_{0}\right) E\left\{\log _{2}\left(1+S_{i k}^{u}\right)\right\}
$$


Where $\mu_{0}$ is the pilot overhead coefficient, indicating the loss of spectral efficiency caused by the pilot sequence during channel estimation. When other pilot scheduling schemes are used, $\mu_{0}$ should be adjusted accordingly.

\section{Scheme of Joint Polar Coordinate Pilot Assignment and Pseudo Random Code Pilot Design}

\subsection{Introduction of the Scheme}

According to Equation (8), the SINR of users in the cell is mainly limited by large-scale fading, which is mainly related to the distance from the user to the target cell base station. Different users in the system have different channel fading because of the different distances. Therefore, there is also a difference in interference effects between different users that multiplex the same pilot sequence. By reasonably arranging the allocation of pilots, the fairness of user services and the overall performance of the system can be effectively improved. In this paper, the influence of pilot contamination on the system is reduced by assigning the pilot sequence reasonably and minimizing the interference between users using the same pilot sequence.

As shown in Figure 1, it is assumed that there are three cells, and each cell randomly distributes six single antenna users.

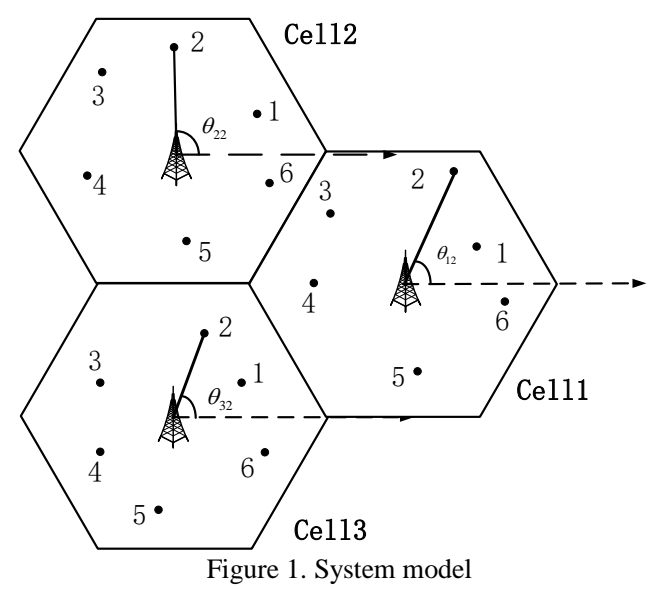

According to the orientation of the client, the polar angles are marked from small to large. The polar angles are $\left\{\theta_{11}, \theta_{12}, \theta_{13}, \theta_{14}, \theta_{15}, \theta_{16}\right\},\left\{\theta_{21}, \theta_{22}, \theta_{23}, \theta_{24}, \theta_{25}, \theta_{26}\right\}$, and $\left\{\theta_{31}, \theta_{32}, \theta_{33}, \theta_{34}, \theta_{35}, \theta_{36}\right\}$ in cell 1 , cell 2 , and cell 3 , respectively. The users in the cell share orthogonal pilot sequences marked $\left\{\varphi_{1}, \varphi_{2}, \varphi_{3}, \varphi_{4}, \varphi_{5}, \varphi_{6}\right\}$.

Through the pilot allocation scheme, the problem that the inter-cell edge users are greatly degraded due to pilot multiplexing is solved. However, interference caused by multiplexing the same pilot sequence between cells still exists.

Then, the pilot sequences of users in the system are artificially scrambled by using pseudo random codes with different delays, thereby reducing the correlation between users of different cells in the same pilot sequence. Therefore, the suppression of pilot pollution is maximized, and the system performance is improved [15].

A pseudo random code is a determined sequence that has characteristics similar to random sequences. It has strong autocorrelation and cross correlation. In this paper, the advantage of the pseudo random code is taken to improve the orthogonality between users assigned to the same pilot sequence in different cells. Under the premise that the cells in the system share the same set of orthogonal pilot sequences, different pseudo random codes with different delays are allocated to different cells, thereby distinguishing different cells, and the pilot sequence of each cell user is interfered to obtain a new pilot sequence. The design scheme of the pseudo random code scramble pilot sequence is shown in Figure 2.

Usually, the pseudo random code is much longer than the pilot sequence. In order to ensure the smooth scrambling of the pilot sequence by pseudo random codes, the pseudo random code is pre-processed. First, generate a rectangular window with the length of the pilot sequence, truncate the generated pseudo random code, and then modulate the truncated pseudo random sequence to make its spectrum shift to the appropriate frequency band. Finally, the modulated pseudo random code is diagonalized. The processed pseudo random code matrix is 


$$
\boldsymbol{P}=\left[\begin{array}{cccc}
m_{1} & 0 & \cdots & 0 \\
0 & m_{2} & \cdots & 0 \\
\vdots & \vdots & \ddots & \vdots \\
0 & 0 & \cdots & m_{\tau}
\end{array}\right]
$$

Substitute the matrix into the scrambler, and the pseudo random sequence of the user after scrambling is obtained.

$$
\psi_{k}=P \phi_{k}=\left[m_{2} \phi_{k 2}, m_{2} \phi_{k 2}, \cdots, m_{\tau} \phi_{k \tau}\right]
$$

In this scheme, the same pseudo random code is used to perform scrambling on all users in the cell, and the pilot sequence processed by $K$ users in the cell is

$$
\Psi=\left[\begin{array}{ccc}
m_{1} \phi_{11} & \cdots & m_{1} \phi_{k 1} \\
\vdots & \ddots & \vdots \\
m_{\tau} \phi_{1 \tau} & \cdots & m_{\tau} \phi_{k \tau}
\end{array}\right]
$$

Thereafter, the user may send the processed pilot sequence to the base station, and the base station will use the sequence for channel estimation to obtain the required channel state information.

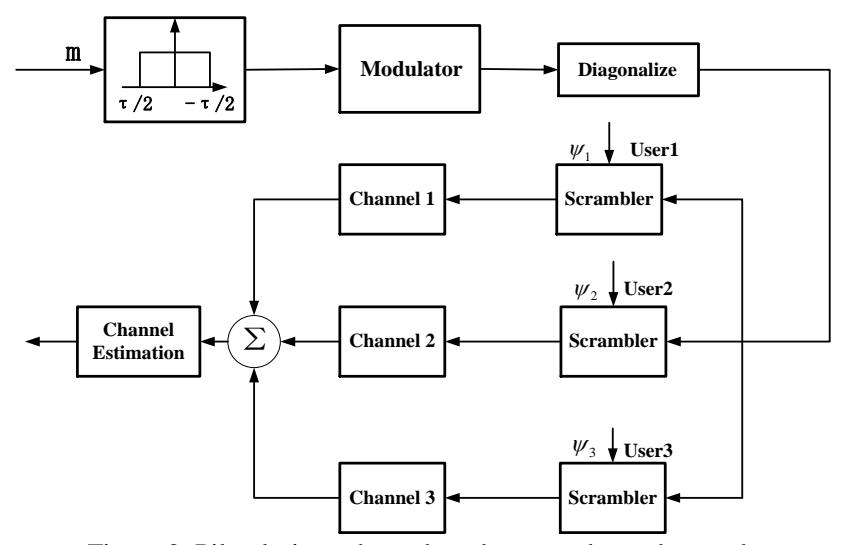

Figure 2. Pilot design scheme based on pseudo random code

In addition, the selection of pseudo random codes is also considered in this scheme because of the different cross correlations between different pseudo random codes. If a pseudo random code with a large cross correlation is selected to process the pilot sequence, the influence of pilot pollution between cells on the system will still be severe. In order to solve this problem, a pseudo random code with less cross correlation should be chosen to process the pilot sequence. According to theoretical analysis, the smaller the cross correlation between different pseudo random codes, the more obvious the effect of pilot pollution suppression. Therefore, a pseudo random code is employed to process the pilot sequence to satisfy

$$
\left|\rho\left(m_{i}, m_{j}\right)\right|_{i \neq j}<\gamma_{i}, \quad j=1,2, \cdots, M
$$

Where $M$ is the number of cells. $\rho\left(m_{i}, m_{j}\right)=\frac{1}{n} \sum_{k=1}^{n} m_{i k} m_{j k}$ is the normalized cross correlation function between different pseudo random codes. $\gamma \in[0,1]$ is a constant that represents the upper limit of the normalized correlation function value between different pseudo random codes used to scramble the pilot sequence.

\subsection{Performance Analysis}

By analyzing the uplink SINR (signal to interference and noise ratio) and the MSE of channel estimation, the performance of the new scheme for reducing pilot contamination is analyzed. 
The uplink SINR of the $k^{\text {th }}$ user in the $i^{\text {th }}$ cell is

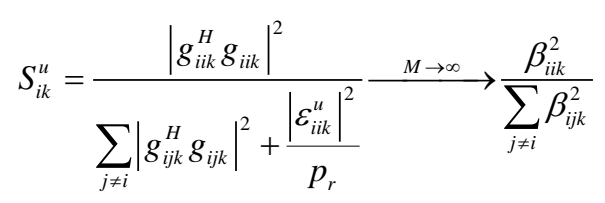

When $M$, the number of antennas of the base station, approaches infinity, the additive noise and small-scale fading will be averaged off. However, in the uplink, the system capacity will be affected by pilot contamination, as shown in Equation (15). Reducing $\sum_{j \neq i} \beta_{i j k}^{2}$ will be the key to reducing pilot contamination and improving the overall performance of the system.

In Equation (2), it can be seen that the large-scale fading in the system is inversely proportional to the distance from the user to the base station. If the distance from user $u_{j k}^{\prime}$ to the base station satisfies $r_{i j k}^{\prime}>r_{i j k}$, then $\beta_{i j k}^{\prime} \propto \frac{1}{r_{i j k}^{\prime}}<\frac{1}{r_{i j k}} \propto \beta_{i j k}$. Therefore, adjusting the distance of users in the same pilot sequence in the system will directly affect the uplink SINR of the user. Thus, increasing the distance between users who are assigned to the same pilot sequence can effectively reduce the impact of pilot pollution on the system.

After the $k^{\text {th }}$ user in the cell is scrambled by the pseudo random code, it can be obtained from Equation (12) where the pilot sequence is $\psi_{k}=P \varphi_{k}$, and then Equation (4) can be expressed as

$$
Y_{i}^{p}=\sqrt{p_{r}} \sum_{j=1}^{L} \sum_{k=1}^{K} g_{i j k} \psi_{k}^{T}+N_{i}^{p}=\sqrt{p_{r}} \sum_{j=1}^{L} \sum_{k=1}^{K} g_{i j k} \varphi_{k}^{T} P_{j}^{T}+N_{i}^{p}
$$

When the number of base station antennas is limited, the MMSE estimate of the desired channel is

$$
\hat{G}_{i i}=\frac{1}{\sqrt{p_{r}}} Y_{i}^{p}\left(C_{n}+p_{r} \tau \sum_{j=1}^{L} \Psi_{i}^{*} C_{i j} \Psi_{i}^{T}\right)^{-1} \Psi_{i}^{*} C_{i i}
$$

Where $C_{n}=E\left\{n_{i}^{H} n_{i}\right\}$ is the autocorrelation matrix of the received noise $n_{i}$, and $C_{i j}=E\left\{G_{i j}^{H} G_{i j}\right\}$ is the autocorrelation matrix of the channel transmission matrix $G_{i j}$.

It can be seen from Equation (5) that the channel estimation result between the $k^{\text {th }}$ user and the cell base station is the channel transmission vectors' superposition of the target cell base station and all users using the same pilot sequence. This is the cause of pilot contamination. In this scheme, the cross correlation value of the multiplexed pilot sequences between different cells is reduced by adding the artificial interference of the pseudo random code to the pilot sequences, which are multiplexed by different cells. Therefore, channel estimation interference caused by pilot contamination is reduced, and then the MSE of the channel estimate is also reduced.

Define the MSE of the channel estimation of the $i^{\text {th }}$ cell as

$$
M^{m s e} \triangleq E\left\{\left\|\hat{G}_{i i}-G_{i i}\right\|_{F}^{2}\right\}
$$

Where $R=\frac{1}{\sqrt{p_{r}}}\left(C_{n}+p_{r} \tau \sum_{j=1}^{L} \Psi_{i}^{*} C_{i j} \Psi_{i}^{T}\right)^{-1} \Psi_{i}^{*} C_{i i}$ and $\hat{G}_{i i}=Y_{i} R$. Substituting it into Equation (18) gives

$$
M^{m s e}=E\left\{\operatorname{tr}\left\{\left(Y_{i} R-G_{i i}\right)^{H}\left(Y_{i} R-G_{i i}\right)\right\}\right\}=\operatorname{tr}\left\{R^{H} E\left\{Y_{i}^{H} Y_{i}\right\} R-R^{H} E\left\{Y_{i}^{H} G_{i i}\right\}-E\left\{G_{i i}{ }^{H} Y_{i}\right\} R+E\left\{G_{i i}{ }^{H} G_{i i}\right\}\right\}
$$

From Equation (16), 


$$
\begin{gathered}
E\left\{Y_{i}^{H} Y_{i}\right\}=C_{n}+p_{r} \tau \sum_{j=1}^{L} \Psi_{i}^{*} C_{i j} \Psi_{i}^{T} \\
E\left\{Y_{i}^{H} G_{i i}\right\}=\sqrt{p_{r} \tau} \Psi_{i}^{*} C_{i i} \\
E\left\{G_{i i}{ }^{H} Y_{i}\right\}=\sqrt{p_{r} \tau} C_{i i} \Psi_{i}^{T}
\end{gathered}
$$

Substituting Equations (20)-(22) into Equation (19) gives

$$
\begin{aligned}
M^{m s e} & =\operatorname{tr}\left\{R^{H}\left(C_{n}+p_{r} \tau \sum_{j=1}^{L} \Psi_{i}^{*} C_{i j} \Psi_{i}^{T}\right)\left(Y_{i} R-G_{i i}\right)^{H} R-\sqrt{p_{r} \tau} R^{H} \Psi_{i}^{*} C_{i i}-\sqrt{p_{r} \tau} C_{i i} \Psi_{i}^{T}+C_{i i}\right\} \\
& =\operatorname{tr}\left\{C_{i i}-\sqrt{p_{r} \tau} C_{i i} \Psi_{i}^{T} R\right\}=\operatorname{tr}\left\{C_{i i}-p_{r} \tau C_{i i} \Psi_{i}^{T}\left(C_{n}+p_{r} \tau \sum_{j=1}^{L} \Psi_{i}^{*} C_{i j} \Psi_{i}^{T}\right)^{-1} \Psi_{i}^{*} C_{i i}\right\}
\end{aligned}
$$

According to the matrix inversion principle,

$$
M^{m s e}=\operatorname{tr}\left\{C_{i i}-p_{r} \tau C_{i i}\left(\Psi_{i}^{T} C_{n} \Psi_{i}^{*}+p_{r} \tau \sum_{j=1}^{L} \Psi_{i}^{T} \Psi_{i}^{*} C_{i j} \Psi_{i}^{T} \Psi_{i}^{*}\right)^{-1} C_{i i}\right\}
$$

When $M \rightarrow \infty$,

$$
\begin{gathered}
C_{i i}=E\left\{G_{i i}{ }^{H} G_{i i}\right\}=\sqrt{B_{i i}} E\left\{H_{i i}{ }^{H} H_{i i}\right\} \sqrt{B_{i i}}=M B_{i i} \\
C_{n}=E\left\{n_{i}^{H} n_{i}\right\}=M I
\end{gathered}
$$

Where $B_{i i}=\left[\beta_{i i 1}, \beta_{i i 2}, \cdots, \beta_{i i K}\right], I \in C^{\tau \times \tau}$, and $H_{i i}{ }^{H}=\left[\begin{array}{ccc}h_{i 1 i 1} & \cdots & h_{i 1 i M} \\ \vdots & \ddots & \vdots \\ h_{i K i K} & \cdots & h_{i K i M}\end{array}\right]$.

Substituting Equations (25)-(26) into Equation (24) gives

$$
\frac{1}{M} M^{m s e}=\operatorname{tr}\left\{B_{i i}-p_{r} \tau B_{i i}\left(I+p_{r} \tau \sum_{j=1}^{L} \Psi_{i}^{T} \Psi_{i}^{*} B_{i j} \Psi_{i}^{T} \Psi_{i}^{*}\right)^{-1} B_{i i}\right\}
$$

When the number of users in each cell $M$ is $1, \Psi_{i}{ }^{T} \Psi_{i}{ }^{*}=\gamma_{i l}$, where $\gamma_{i l}$ is the normalized cross correlation between the pseudo random sequence in the $i^{\text {th }}$ cell and the pseudo random sequence in the $j^{\text {th }}$ cell. Then, Equation (27) can be replaced by

$$
\frac{1}{M} M^{m s e}=\beta_{i i 1}-\frac{p_{r} \tau \beta_{i i 1}^{2}}{1+p_{r} \tau \beta_{i i 1}+p_{r} \tau \gamma_{i j}^{2} \beta_{j i 1}}
$$

It can be obtained from Equation (28) that the MSE obtained in this scheme is mainly limited by the cross correlation between the pseudo random sequences used, while the large-scale fading coefficient, uplink transmission power, and pilot length are constants. It can be concluded that by selecting a suitable pseudo random sequence, this scheme can effectively improve the performance of channel estimation, thereby reducing pilot contamination.

\section{Simulation Results}

The improvement of the performance of the massive MIMO system is evaluated by means of numerical simulation in this section. The impact on the system performance from adjusting parameters is analyzed. 
In the numerical simulation, three cells are considered for simplifying the calculations. The radius of each hexagonal cell is $1000 \mathrm{~m}$, and each cell contains six users. The average transmit power of the user is $p_{\mathrm{r}}=10 \mathrm{~dB}$, the upper limit of the normalized cross correlation value of the pseudorandom code is $\gamma=0.3$, the logarithmic shadow of the channel is normal fading $z=4 \mathrm{~dB}$, the path loss factor is $\alpha=3$, and the length of pilot sequence is $\tau=64$. In order to reflect the improvement of the system performance of this solution more intuitively, no pilot contamination and no pilot decontamination scheme are included in the simulation.

The channel estimation MSE curves for the number of system antennas between 50 and 500 are shown in Figure 3.

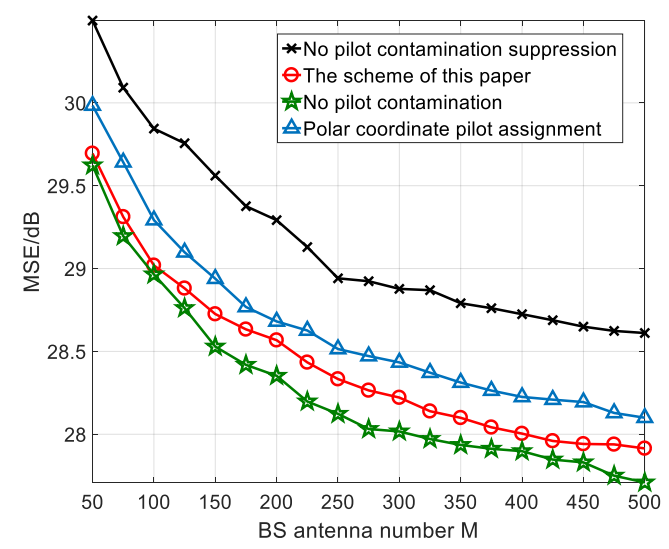

Figure 3. The channel estimation MSE curves

The scheme of only using polar coordinate pilot assignment and the scheme of joint polar coordinate pilot assignment and pseudo random code pilot design adopted in this paper demonstrate good performances in channel estimation compared with the scheme without pilot contamination suppression. The value of the MSE is increased by nearly $1 \mathrm{~dB}$. However, the scheme adopted in this paper can be further improved in performance compared with that of only using polar coordinate pilot assignment. Therefore, the system performance does not increase the algorithm complexity. In the case, it is closer to the state when there is no pilot contamination.

Figure 4 shows the cumulative distribution function (CDF) of the uplink SINR of the target cell user in different conditions when the number of cell base station antennas is $M=1000$. It can be seen that the SINR of the scheme in this paper is effectively improved compared with the polar coordinate pilot assignment scheme, and the SINR of the uplink is effectively improved by about $10 \mathrm{~dB}$. The system performance is closer to the situation without pilot contamination, and the influence of pilot contamination on system performance is greatly suppressed.

Figure 5 shows the relationship between the average reachable rate of the uplink user terminal of the target cell in the system and the number of base station antennas in different conditions. It can be seen that the average reachable rate of the scheme in this paper has a significant improvement compared with that of the polar coordinate pilot assignment scheme. The reachable rate is increased by nearly $3 \mathrm{bps} / \mathrm{Hz}$. It is closer to the average reachable rate without pilot contamination.

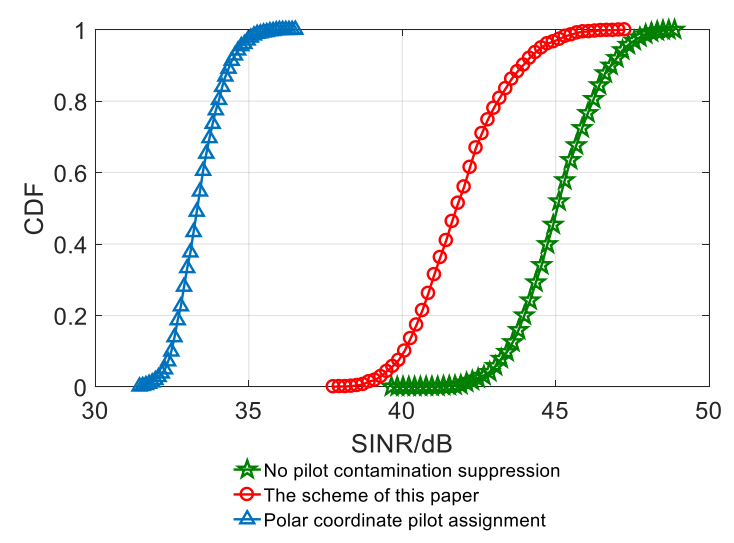

Figure 4. The cumulative distribution function of the SINR

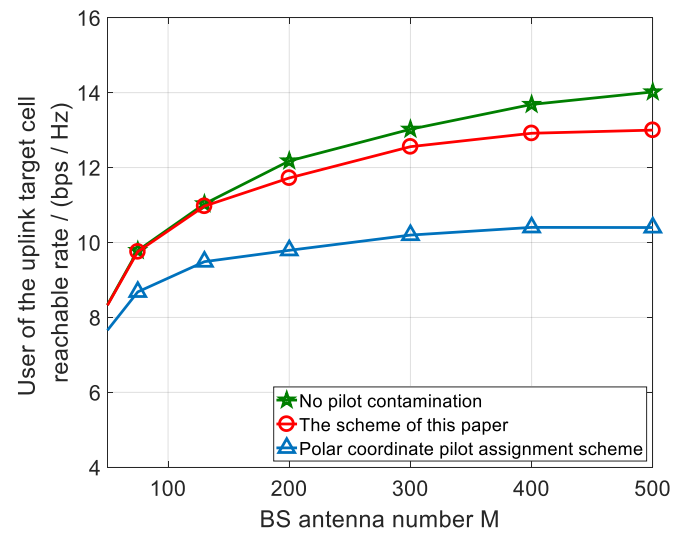

Figure 5. The average client reachable rate of uplink 


\section{Conclusions}

In order to suppress the pilot contamination problem in massive MIMO systems, a scheme of joint polar coordinate pilot assignment and pseudo random code pilot design is proposed in this paper. The specific implementation steps of this scheme are introduced, and the theoretical derivation and data simulation of performance indicators are carried out. Through the simulation of the MSE of the channel estimation in the system, the CDF of the uplink SINR of the target cell, and the average reachable rate of the uplink user of the target cell, the scheme is compared with the situation of no pilot contamination and the scheme based on polar coordinate pilot assignment. The overall performance of the system has been significantly improved.

\section{Acknowledgements}

The authors would like to thank the Higher Educational Key Laboratory for Signal \& Information Processing of Heilongjiang Province. This work is financially supported by the National Natural Science Foundation of China (No. 61601149) and Science Foundation of Heilongjiang Province (No. QC2017074).

\section{References}

1. X. You, Z. Pan, and X. Gao, "The 5G Mobile Communication: The Development Trends and Its Emerging Key Techniques," Scientia Sinica Information, Vol. 44, No. 5, pp. 551-563, May 2014

2. T. Marzetta, "Noncooperative Cellular Wireless with Unlimited Numbers of Base Station Antennas," IEEE Transactions on Wireless Communications, Vol. 9, No. 11, pp. 3590-3600, November 2010

3. Y. Lin, X. Zhu, and Z. Zheng, "The Individual Identification Method of Wireless Device based on Dimensionality Reduction and Machine Learning," Journal of Supercomputing, No. 5, pp. 1-18, May 2017

4. Y. Lin, C. Wang, J. X. Wang, and Z. Dou, “A Novel Dynamic Spectrum Access Framework based on Reinforcement Learning for Cognitive Radio Sensor Networks," Sensors, Vol. 16, No. 10, pp. 1-22, October 2016

5. Z. Y. Zhang, X. H. Guo, and Y. Lin, "Trust Management Method of D2D Communication based on RF Fingerprint Identification," IEEE Access, No. 6, pp. 66082-66087, June 2018

6. S. Albdran, A. Alshammari, and M. Ahad, "Effect of Exponential Correlation Model on Channel Estimation for Massive MIMO," in Proceedings of IEEE International Conference on Computer and Information Technology, pp. 80-83, Dhaka, Bangladesh: IEEE Press, December 2016

7. Q. Wang and C. Qiu, "Subspace-based Semi-Blind Channel Estimation for Massive MIMO Systems," Computer Engineering and Applications, Vol. 54, No. 8, pp. 91-95, August 2018

8. Y. Zhang, R. Venkatesan, and O. Dobre, "Novel Compressed Sensing-based Channel Estimation Algorithm and Near-Optimal Pilot Placement Scheme," IEEE Transactions on Wireless Communications, Vol. 15, No. 4, pp. 2590-2603, April 2016

9. Q. Zhou, K. Xu, and Y. Xu, "Joint Beam Domain Decomposition and SVD for Channel Estimation in Multi-User Massive MIMO System," Journal of Signal Processing, Vol. 43, No. 4, pp. 439-447, April 2018

10. X. Gao, L. Dai, and J. Zhang, "Capacity-Approaching Linear Precoding with Low-Complexity for Large-Scale MIMO Systems," in Proceedings of 2015 IEEE International Conference on Communications, pp. 1577-1582, London, UK: IEEE Press, June 2015

11. Y. Wu, T. Liu, and M. Cao, "Pilot Contamination Suppression in Massive MIMO Multi-Cell Multi-User TDD Systems," Advanced Engineering Sciences, Vol. 50, No. 2, pp. 105-111, February 2018

12. Q. Yang, Q. Wang, and F. Niu, "Research on ZC Code Pilot Design in Massive MIMO," Information and Communications, Vol. 184, No. 4, pp. 1-3, April 2018

13. Y. Tu, Y. Lin, and J. Wang, "Semi-Supervised Learning with Generative Adversarial Networks on Digital Signal Modulation Classification," CMC-Computers Materials and Continua, Vol. 55, No. 2, pp. 243-254, February 2018

14. Y. Lin, Y. Li, and X. Yin, "Multisensor Fault Diagnosis Modeling based on the Evidence Theory," IEEE Transactions on Reliability, Vol. 67, No. 2, pp. 513-521, February 2018

15. X. Fang, J. Zhang, and H. Cao, "Pilot Design based on Pseudo-Random Code in Massive MIMO System," Journal of Applied Sciences-Electronics and Information Engineering, No. 1, pp. 1-11, January 2016 